positive for ANCA (SLE, sclerosis, RA, RA/RV). This study shows that the PR3and MPO-ANCA ELISA are highly specific (93.2\%/94.2\%) and sensitive (85.9\%/ $85.9 \%$ ) in the detection of ANCA to identify AAV or conditions known to be associated ANCA.

Conclusions: Our comparison of PR3- and MPO-ANCA ELISAs showed (i) a high diagnostic performance of these PR3- and MPO-ANCA ELISAs to discriminate AAV from disease controls. (ii) very good correlation between the other methods tested. In conclusion, these novel assays can be used as screening method for detection of ANCA-associated diseases.

Disclosure of Interest: None declared

DOI: 10.1136/annrheumdis-2018-eular.6352

\section{FRI0651 QUANTITATIVE CHEST CT PREDICTS 8-YEARS- MORTALITY AND COMORBIDITY IN SYSTEMIC SCLEROSIS}

A. Ariani ${ }^{1}$, M. Silva ${ }^{2}$, E. Bravi ${ }^{3}$, M. Saracco ${ }^{4}$, S. Parisi ${ }^{5}$, F. De Gennaro ${ }^{6}$, L. Idolazzi ${ }^{7}$, M. De Santis ${ }^{8}$, F. Girelli ${ }^{9}$, F. Lumetti ${ }^{10}$, A. Volpe ${ }^{11}$, D. Santilli', F. Mozzani ${ }^{1}$, G. Lucchini ${ }^{1}$, D. Giuggioli ${ }^{12}$, V. Seletti ${ }^{13}$, E. Bacchini ${ }^{13}$, C. Benini ${ }^{7}$, E. Arrigoni ${ }^{3}$, F. C. Bodini ${ }^{14}$, G. Paolazzi ${ }^{11}$, D. Imberti ${ }^{3}$, E. Fusaro ${ }^{5}$, C. A. Scirè ${ }^{15}$, N. Sverzellati ${ }^{16}$. ${ }^{1}$ Department of Medicine, Internal Medicine and Rheumatology Unit, Azienda Ospedaliero-Universitaria di Parma, ${ }^{2}$ Department of Clinical Sciences, Section of Radiology, University of Parma, Parma, ${ }^{3}$ Department of Medicine, Internal Medicine and Rheumatology Unit, Ospedale G. Da Saliceto, Piacenza, ${ }^{4}$ Rheumatology Unit, Ospedale Mauriziano Umberto I di Torino, ${ }^{5}$ Rheumatology Department, Azienda Ospedaliera Città della Salute e della Scienza di Torino, Torino, ${ }^{6}$ Rheumatology Unit, Azienda Ospedaliera Istituti Ospitalieri di Cremona, Cremona, ${ }^{7}$ Rheumatology Section, Department of Medicine, University of Verona, Azienda Ospedaliero Universitaria Integrata, Verona, ${ }^{8}$ Rheumatology and Clinical Immunology, Humanitas Research Hospital, Milano, ${ }^{9}$ Rheumatology Unit, Internal Medicine Department, GB Morgagni - L Pierantoni Hospital, Forli, ${ }^{10}$ Rheumatology Unit., University of Modena and Reggio Emilia, Azienda Ospedaliero- Universitaria, Policlinico di Modena, Modena, ${ }^{11}$ Rheumatology Unit, Santa Chiara Hospital, Trento, ${ }^{12}$ Rheumatology Unit, University of Modena and Reggio Emilia, Azienda Ospedaliero- Universitaria, Policlinico di Modena, Modena, ${ }^{13}$ Department of Clinical Sciences, Section of Radiology, University of Parma, Parma, ${ }^{14}$ Radiology Unit, Ospedale G. Da Saliceto, Piacenza, ${ }^{15}$ Department of Medical Sciences, Section of Rheumatology, University of Ferrara and AOU Sant'Anna, Ferrara, ${ }^{16}$ Department of Clinical Sciences, Section of Radiology, University of Parma, Parma, Italy

Background: Interstitial Lung Disease (ILD) is the main cause of death in Systemic Sclerosis (SSc). Serious lung involvement increase the hospitalization rate and sometimes oxygen therapy is required.

An prompt treatment in ILD-SSc patients is essential, but nowadays there is not a feasible method to identify patients with poor prognosis.

Although chest Computed Tomography (CT) is the gold standard to detect ILD, there is no standardized assessment of its severity. Quantitative CT (QCT) is an innovative and operator independent method to assess ILD-SSc extent and severity. An increasing number of evidences confirm that QCT is extremely useful for detecting SSc-ILD with the worst prognosis. However is not well established if QCT can predict death or comorbidity associated to ILD.

Objectives: The main aim of this study is to verify if QCT predict 8-years mortality or clinical worsening (i.e. hospitalization for respiratory complications or chronic oxygen therapy) in SSc

Methods: Consecutive SSc patients (according to ACR/EULAR classification criteria) from ten different centers underwent a chest $C T$. Their clinical history was carefully recorded in the following 96 months. A rheumatologist post-analyzed every CT with an open source DICOM viewer in order to obtain QCT indexes. Patients were clustered in two subgroups: according to QCT indexes' cutoff previously found (see Ariani et al., 2017). The survival time of each patient was considered the time interval between the CT date and death, hospitalization related to lung worsening, beginning of chronic oxygen therapy or last medical examination Results: We enrolled 342 patients; during the 8-years follow-up 15.5\% (53/342) died and 4,4\% (15/342) had a clinical worsening. The Kaplan-Meier survival analysis demonstrates a worse survival in patients with QCT indexes above or below the previously observed cutoffs. ( $<<0.01)$. In particular the QCT index cutoff which the best performance was total lung kurtosis (tKurt) equal to $4.89(p<0.0001)$.

\section{REFERENCES:}

The QCT indexes' cutoffs previously found (see Ariani, et al., 2017) identify the SSc-ILD subjects with an high risk of 8-years mortality and comorbidity. These findings suggest that QCT could became a pivotal assessment in SSc management because its role in identifying patients with poor prognosis and who deserve an early aggressive treatment.

Disclosure of Interest: None declared
DOI: 10.1136/annrheumdis-2018-eular.5709

\section{FRI0652 THE DEVELOPMENT AND VALIDATION OF FAMILIAL MEDITERRENEAN FEVER QUALITY OF LIFE SCALE (FMF-QOL)}

C. Unal ${ }^{1}$, M. T. Duruoz ${ }^{2} .{ }^{1} P M R$ Department, ${ }^{2} P M R$ Department, Rheumatology Division, Marmara University School of Medicine, Istanbul, Turkey

Background: Quality of life (QoL) was negatively affected in FMF patients. A QoL scale specific to FMF is not existing in the literature.

Objectives: To develop valid and reliable quality of life scale in familial Mediterranean fever (FMF).

Methods: The psychometric method was used to develop the FMF-QoL. In the first step, the question pool was formed by using existing QoL scales in the literature. Outpatients with FMF according to Livneh criteria were recruited. After patients' interviews, some identical and irrelevant questions eliminated and new ones were added by an expert committee. Cognitive debriefing interviews concerning QoL were done with another 20 FMF patients. Minor changes (few words) after these interviews were made. In the second step, the preFMF-QoL with 101 questions was formed and it was filled out by FMF patients. Confirmatory factor analysis (CFA) by varimax rotation, assessment of data' skewness and kurtosis, evaluation of invalid questions and participants were performed. After this, the internal consistency with Cronbach alpha was calculated. The face, content and construct validities were assessed. Convergent validity which was the relation of the FMF-QoL with functional parameters (Europe Health Impact Scale (EURO HIS), Short Form 36 (SF36), Beck Depression Inventory for Primary Care (BDIPC), Jenkins Sleep Scale (JSS), Health Assessment Questionnaire (HAQ) and the discriminant validity which was the relation of the FMF-QoL with nonfunctional parameters such as demographic and clinical characteristics were analyzed. The Mann Whitney U test, Kruskal-Wallis test and Spearman correlation coefficient (rho) were used to compare quantitative variables.

Results: In our study, the FMF-QoL was applied to 125 FMF patients however 123 (84 women) patients were designed to statistical analysis. It has been determined by the expert committee that the face and content validity of the FMF-QoL was good. According to the factor analysis the FMF-QoL were represented by 4 factor groups (eigenvalues $>1$ ) which were physical impact, social and recreational impact, psychological impact, and sleep impact factors. All question's factor loadings after Varimax rotation were bigger than 0.5 and the cumulative variance of the scale was $68.11 \%$. The strongest correlation of the FMF-QoL was found with other QoL scales like EUROHIS (rho: -0.64, p<0.01) and SF36-physical functioning subscale (rho: $-0.63, \mathrm{p}<0.01$ ). The correlations between the FMF-QoL and other functional parameters were found to be moderate [BDI-PC (rho: 0.46, $p<0.01$ ), JSS (rho: $0.44, p<0.01$ ), HAQ (rho: $0.44, p<0.01$ )]. This shows that the FMF-QoL has good convergent validity. In the discriminative validity, most of the parameters had not significant correlation with the FMF-QoL. Only the number of attacks in the previous year, age and education level were correlated moderately with the FMF-QoL. This shows the FMF-QoL has discriminative validity.

Conclusions: The FMF-QoL scale was developed with psychometric method. It is a valid and reliable disease specific scale which has 20 questions with 4 factors. It is already a practical, not time consuming that can be used in the clinical follow up and the treatment evaluation.

Disclosure of Interest: None declared

DOI: 10.1136/annrheumdis-2018-eular.6838

\section{FRI0653 \\ VALIDITY OF POLYMYALGIA RHEUMATICA DIAGNOSES, AND CLASSIFICATION CRITERIA, IN} PRIMARY HEALTH CARE

C. Fors ${ }^{1,2}$, U. Bergström ${ }^{1,2}$, M. Willim ${ }^{2,3}$, E. Pilman ${ }^{4}$, C. Turesson $^{1,2}$.

${ }^{1}$ Rheumatology, Department of Clinical Sciences, Malmö, Lund University, ${ }^{2}$ Department of Rheumatology, Skåne University Hospital, Malmö, ${ }^{3}$ Rheumatology, Department of Clinical Sciences, Lund, Lund University, Lund, ${ }^{4}$ Landborgen Primary Health Care Centre, Helsingborg, Sweden

Background: Polymyalgia rheumatica (PMR) is an inflammatory disorder that mainly affects elderly women, and usually is diagnosed in primary health care (PHC). A number of classification criteria have been proposed, most recently the American College of Rheumatology (ACR)/ European League Against Rheumatism (EULAR) criteria from 2012 (1). The ACR/EULAR criteria were developed in a cohort of patients recruited from rheumatology clinics.

Objectives: To examine the validity of PMR diagnoses in primary care, and to validate the use of classification criteria for PMR in a retrospective survey of a PHC cohort.

Methods: Patients were recruited from two PHC centers. All patients with a reg istered diagnosis of PMR between 2000 and 2013 in the patient administrative system were identified. The electronic case records, including hospital records, 